\title{
Angiosarcoma of the Left Atrium: A Case Report
}

\author{
Georg Schlachtenberger ${ }^{1}$ Stephen Gerfer ${ }^{1} \quad$ Axel Kröner $^{1}$ Thorsten Wahlers ${ }^{1}$ \\ ${ }^{1}$ Klinik und Poliklinik fur Herz- und Thoraxchirurgie, Klinikum der \\ Universitat zu Köln, Köln, Germany \\ Thorac Cardiovasc Surg Rep 2018;7:e4-e6.

\begin{abstract}
Address for correspondence Georg Schlachtenberger, Klinik und Koln, Kerpenerstraße 62 Köln, Köln, Nordrhein-Westfalen 50937, Germany (e-mail: Georg.Schlachtenberger@uk-koeln.de).
\end{abstract} \\ Poliklinik fur Herz- und Thoraxchirurgie, Klinikum der Universitat zu
}

\begin{abstract}
Background Primary cardiac tumors are rare, and many diagnosed tumors are benign with an incidence of $0.001 \%$ to $0.03 \%$. The primary angiosarcoma is one of the malignant entities.

Discussion We discuss a case report of a 76-year-old male who underwent a preoperative diagnosis for an upcoming shoulder operation when his cardiologist diagnosed a large cardiac tumor. The patient was referred to our department where he received further diagnostics. The transesophageal echocardiography and the cardiac-magnetic resonance imaging showed a massive tumor with a dimension of $8.6 \times 5.6 \mathrm{~cm}$ with no signs of malignity.

Method The operation was performed by standard median sternotomy. The tumor was adherent to the septum and the left atrium, and we were able to remove the specimen in toto. Pathological examinations showed an angiosarcoma with neovascularization and core expression of ERG + and cytoplasmic expression of CD31+/CD34+, due to the size of the mass. The resection of primary cardiac tumors is mostly the

Keywords

- sarcoma

- myxoma therapy of choice, but in this case concerning an angiosarcoma the prognosis is poor, considering that the angiosarcoma responds very badly to chemotherapy and radiotherapy.
\end{abstract}

\section{Introduction}

A 76-year-old male was found to have a first-degree atrioventricular block and lower leg edema during a preoperative checkup for an upcoming elective shoulder operation. For further diagnostics, the patient underwent a transthoracic echocardiography by his cardiologist which showed a suspected tumor in the left atrium. Thus, the patient was referred to our university hospital for further investigation. The performed transthoracic and transesophageal echocardiography ( - Fig. 1) as well as the cardiac-magnetic resonance imaging (c-MRI) confirmed a suspected tumor with a dimension of $8.6 \times 5.6 \mathrm{~cm}(-$ Fig. 2 ). The $\mathrm{c}-$ MRI showed a hypointense tumor mass inside the left atrium attached to the septum with an inhomogeneous peripheral enrichment of contrast agent with no proof of infiltration. This tumor is most likely compatible with a myxoma with no indication for a sarcoma in the imaging.

received

November 14, 2017

accepted after revision

December 18, 2017
DOI https://doi.org/

10.1055/s-0038-1627443. ISSN 2194-7635.
The operation was performed by a standard median sternotomy and cannulation of the aorta and the venae cava superior and inferior. Cardiopulmonary bypass time was 193 minutes, the aortic cross clamp time was 107 minutes, and the reperfusion time was 83 minutes. As cardioprotection, $450 \mathrm{~mL}$ of cold Buckberg cardioplegia was used. In addition, the patient received a coronary artery bypass graft on his left artery descending using the left internal thoracic artery.

The left atrium was approached through a transseptal incision coming from the right atrium. The removal of the tumor was rather complex because it was adherent to the atrial septum and the roof of the left atrium and ranged from the aorta to the coronary sinus.

It was required to dissect the left atrial roof, due to the tumor extension. The tumor was adherent to the coronary sinus, so the tumor was dissected carefully from that vessel

\footnotetext{
(c) 2018 Georg Thieme Verlag KG Stuttgart · New York
}

License terms

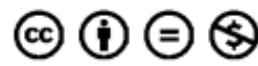




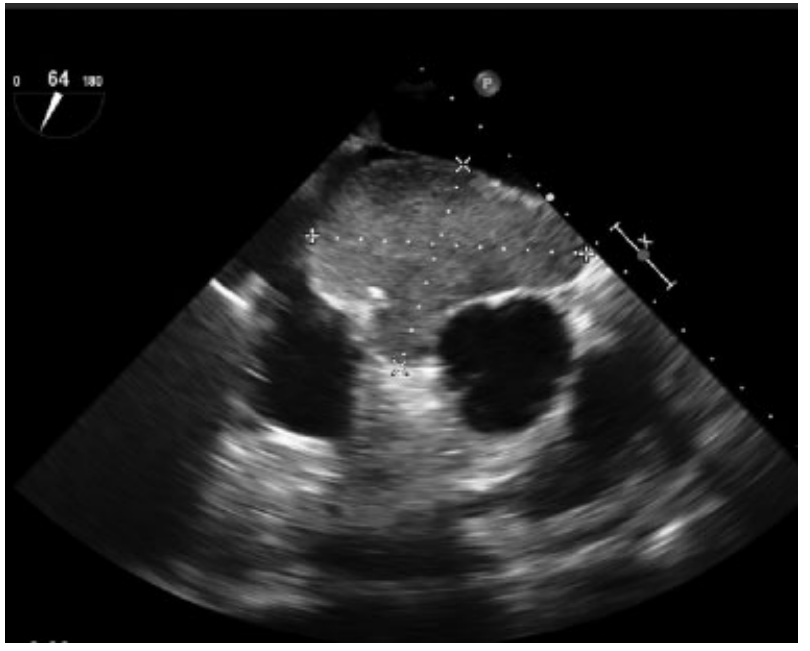

Fig. 1 Transesophageal echocardiography showing a tumor close to the left atrium.

only to leave a thin membrane covering the sinus. Furthermore, the tumor was dissected from the left auricule to the anterior leaflet of the mitral valve to make sure it was resected properly.

Lastly, it was followed by the removal of the caudal part of the tumor mass from the coronary sinus through the triangle of Koch with only $5 \mathrm{~mm}$ distance to the tricuspid valve dissecting through the atrial septum. With this technique, we dissected the tumor in toto.

The left atrial roof and the septum were reconstructed by an Auto-Tissue-Berlin-Matrix Patch using a Prolene 4.0 suture. A total atrioventricular block was unavoidable attributable to the sutures alongside the coronary sinus, tricuspid valve, aortic valve, and the anterior leaflet of the mitral valve. Therefore, our patient received an epimyocardial dual chamber pacemaker (Boston Scientific Essentio) in the same session. The specimen looked nothing like a myxoma sowing intratumoral hemorrhaging (-Figs. 3 and $\mathbf{4}$ ).

Later, the probe was sent to our pathology department for further investigations. The pathological expertise showed an

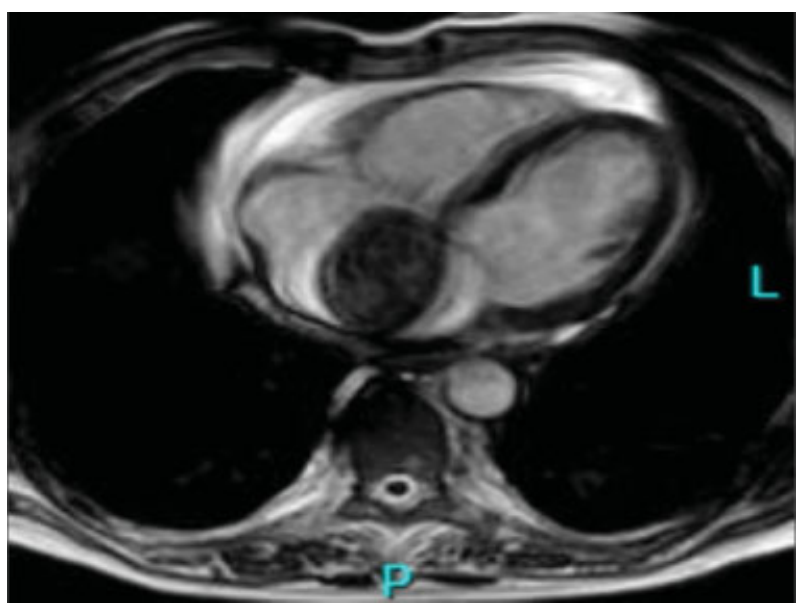

Fig. 2 A cardiac magnetic resonance imaging scan showing the tumor mass.

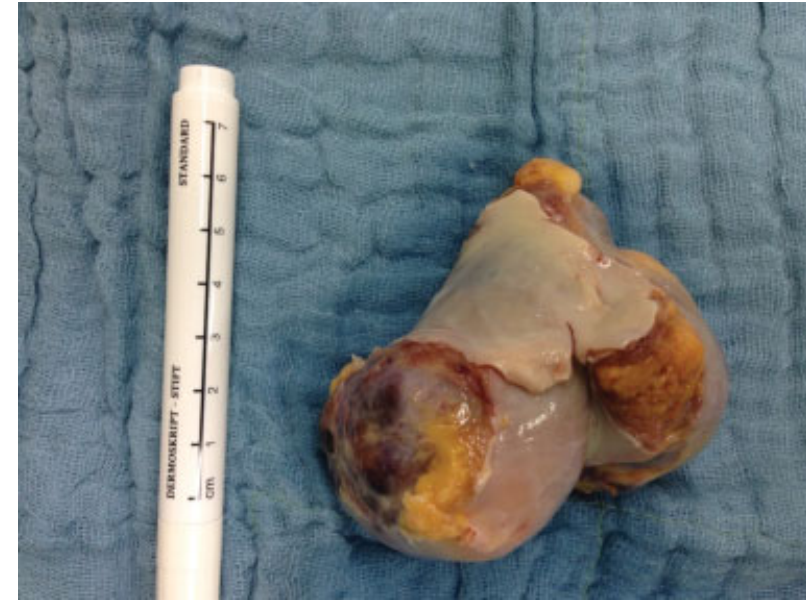

Fig. 3 Removed tumor mass.

angiosarcoma with neovascularization and core expression of ERG + , cytoplasmic expressions of CD31 + /CD34 + , Kras, and $\mathrm{N}$-ras wild type without any mutation in the examined ras-gene.

After the operation, the patient was sent to our intensive care unit, where he had an unproblematic postoperative course. Due to the advanced age of the patient and the bad response to chemotherapy, the department of oncology decided to only perform a tangential radiation therapy. Seven months after the resection of the tumor, the patient is still under active surveillance with good quality of life.

\section{Discussion}

Primary heart tumors are rare. The frequency of cardiac tumors is described with $0.001 \%$ to $0.03 \%$. ${ }^{1}$ About one quarter of these tumors have a malignant entity. ${ }^{2}$ Most malignant heart tumors are sarcomas, but they are still extremely rare. Patients with primary cardiac tumors usually remain clinically silent until they reach an advanced stage. Thus, they are

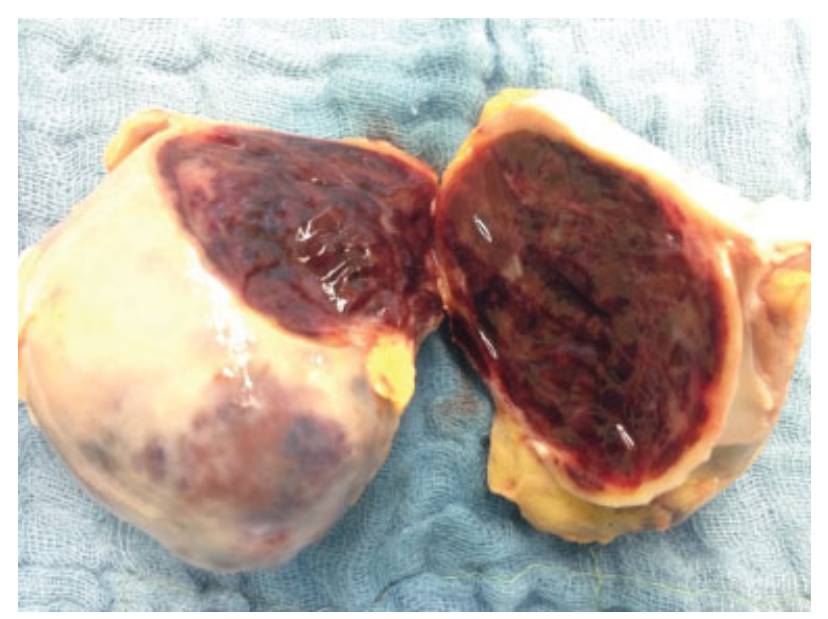

Fig. 4 Removed tumor mass. 
e6 Angiosarcoma of the Left Atrium Schlachtenberger et al.

often found accidentally by echocardiography when they cause clinical symptoms like described in our case report. The evaluation of an intracardial tumor is complex and should include a variety of diagnostics as performed in our case. $^{3}$

The resection of the tumor was successful, and the patient was able to leave our department in a good constitution receiving a tangential therapy in progress. The prognosis is, however, still poor considering that the tumor responds badly to chemo- and radiotherapy. ${ }^{4}$

\section{References}

1 Reynen K. Frequency of primary tumors of the heart. Am J Cardiol 1996;77(01):107

2 Simpson L, Kumar SK, Okuno SH, et al. Malignant primary cardiac tumors: review of a single institution experience. Cancer 2008; 112(11):2440-2446

3 Campanile A, Tavazzi G, Alam MH, Paul R, Price S. An unexpected finding in an asymptomatic patient with atrial fibrillation: cardiac angiosarcoma. Lancet 2016;387(10036):e29

4 Ma G-T, LiuJ-Z, Miao Q, Cui Q-C. Angiosarcoma of the pericardium: a case report. Int J Clin Exp Pathol 2015;8(10):13568-13570 